\title{
Urea and Urea Nitrate Decomposition Pathways: A Quantum Chemistry Study
}

\author{
Igor V. Tokmakov ${ }^{\mathrm{a}}$, Saman Alavi ${ }^{\mathrm{b}}$, and Donald L. Thompson ${ }^{\mathrm{a}, *}$
}

Supporting Information

TABLE S1. Optimized Geometries (Cartesian Coordinates/ $\AA$ ) of the Species and TS Involved in Urea Isomerization/Decomposition.

\begin{tabular}{|c|c|c|}
\hline & MP2/6-311++G(d,p) & B3LYP/6-311++G(d,p) \\
\hline \multirow{8}{*}{ (1) } & $\begin{array}{llll}\text { C } & 0.00000 & 0.00000 & 0.14811\end{array}$ & $\begin{array}{llll}\text { C } & 0.00000 & 0.00000 & 0.14341\end{array}$ \\
\hline & O $\quad \begin{array}{llll}0.00000 & 0.00000 & 1.36601\end{array}$ & $\begin{array}{llll}O & 0.00000 & 0.00000 & 1.36010\end{array}$ \\
\hline & N $0.000001 .16058-0.61870$ & N $0.000001 .16351-0.60977$ \\
\hline & H -0.22418 $1.98166-0.07508$ & Н -0.19411 $1.99448-0.07190$ \\
\hline & H -0.48645 $1.11183-1.50243$ & H -0.41263 $1.14468-1.53036$ \\
\hline & N $0.00000-1.16058-0.61870$ & N $0.00000-1.16351-0.60977$ \\
\hline & Н $0.48645-1.11183-1.50243$ & Н $0.41263-1.14468-1.53036$ \\
\hline & Н $0.22418-1.98166-0.07508$ & Н $0.19411-1.99448-0.07190$ \\
\hline \multirow{8}{*}{ (2a) } & C -0.055620.090490.00075 & C -0.05192 $0.09309-0.00181$ \\
\hline & O - $0.66713-1.118790 .00690$ & O -0.69581 -1.10205 0.00583 \\
\hline & N $1.31537-0.07399-0.08425$ & N $1.30931-0.10287-0.06697$ \\
\hline & H $1.64845-0.974970 .22807$ & H $1.64651-1.021220 .17843$ \\
\hline & H 1.861830 .702810 .25460 & H 1.898900 .665590 .20663 \\
\hline & N-0.75435 1.161860 .01009 & N -0.72266 1.175100 .00866 \\
\hline & H -0.15475 $1.98331-0.01456$ & H -0.13407 $2.00290-0.01162$ \\
\hline & H -1.61194 -0.91887 -0.00860 & H -1.63992 -0.89502 -0.00107 \\
\hline \multirow{8}{*}{ (2b) } & C $0.05288-0.09639-0.00213$ & C $0.05007-0.10022-0.00382$ \\
\hline & O $0.531201 .18611-0.00578$ & O $0.541581 .18112-0.00432$ \\
\hline & $\mathrm{N}-1.32093-0.06861-0.08122$ & N -1.31814 -0.06302 -0.06481 \\
\hline & H -1.75794 0.746650 .32160 & H -1.77322 $0.77687 \quad 0.25436$ \\
\hline & H -1.75174 -0.94785 0.16206 & H -1.78110 -0.93548 0.13135 \\
\hline & N $0.71185-1.192860 .01616$ & N $0.70486-1.191840 .01421$ \\
\hline & Н $1.71498-1.02194-0.01290$ & H $1.71130-1.05362-0.01089$ \\
\hline & Н 1.491361 .142910 .04361 & Н 1.502981 .148590 .03684 \\
\hline \multirow{8}{*}{$(2 c)$} & C -0.09927 -0.08578 0.00485 & C -0.09617 $-0.09076 \quad 0.00419$ \\
\hline & O -0.40062 1.249240 .04200 & O -0.43450 $1.23742 \quad 0.03711$ \\
\hline & N $1.26981-0.33539-0.08328$ & N $1.27491-0.31042-0.07868$ \\
\hline & Н 1.819130 .174380 .59775 & H $1.83867 \quad 0.233730 .56184$ \\
\hline & Н $1.45924-1.32890-0.04594$ & H $1.50285-1.29511-0.05018$ \\
\hline & N -0.96922 -1.01436 0.01688 & N -0.94241 -1.03098 0.01819 \\
\hline & H -1.89656 -0.59710 -0.05163 & H -1.88893 -0.66400 -0.04992 \\
\hline & Н $0.314601 .72065-0.40042$ & Н $0.272921 .76037-0.36028$ \\
\hline
\end{tabular}




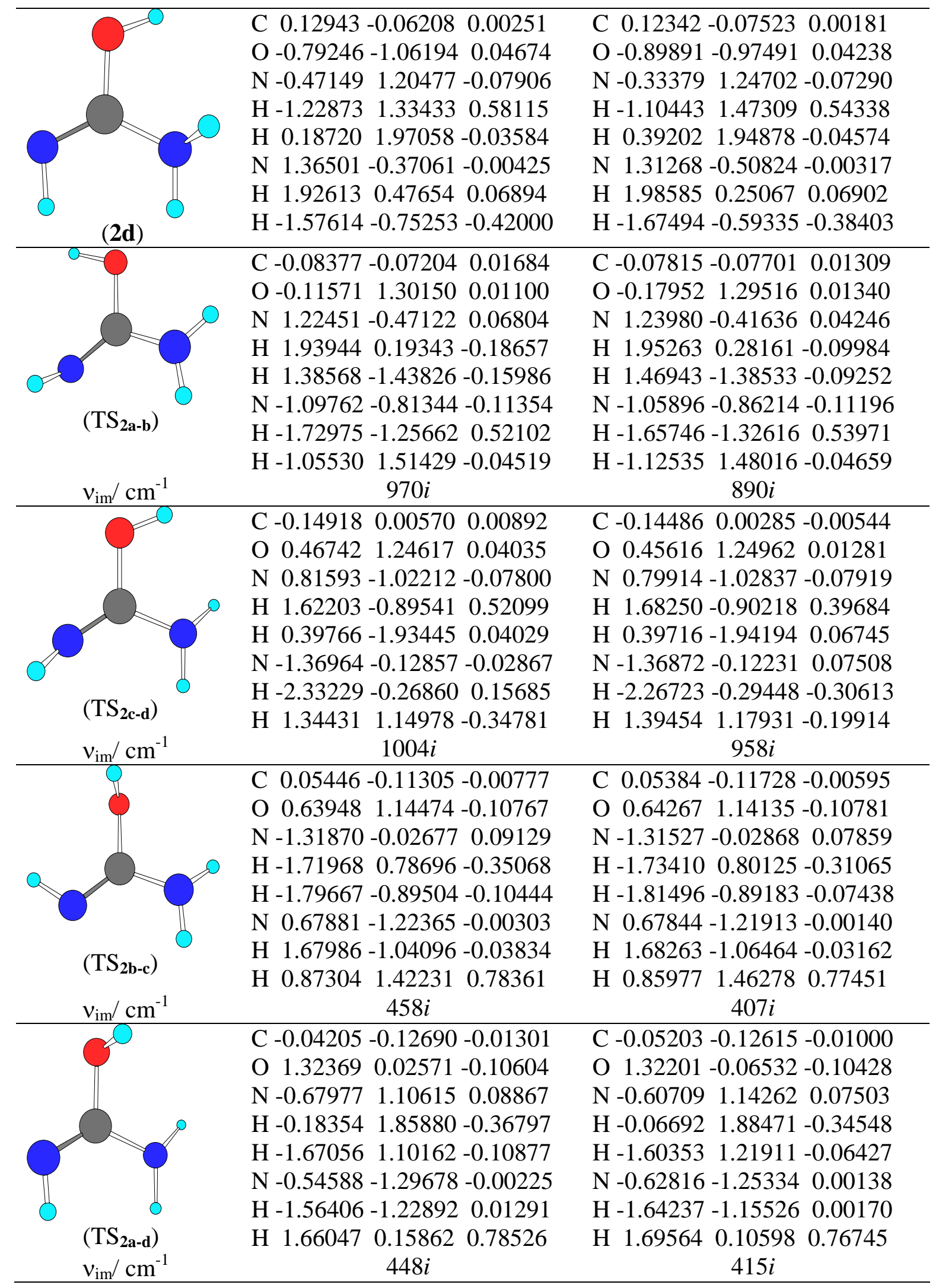




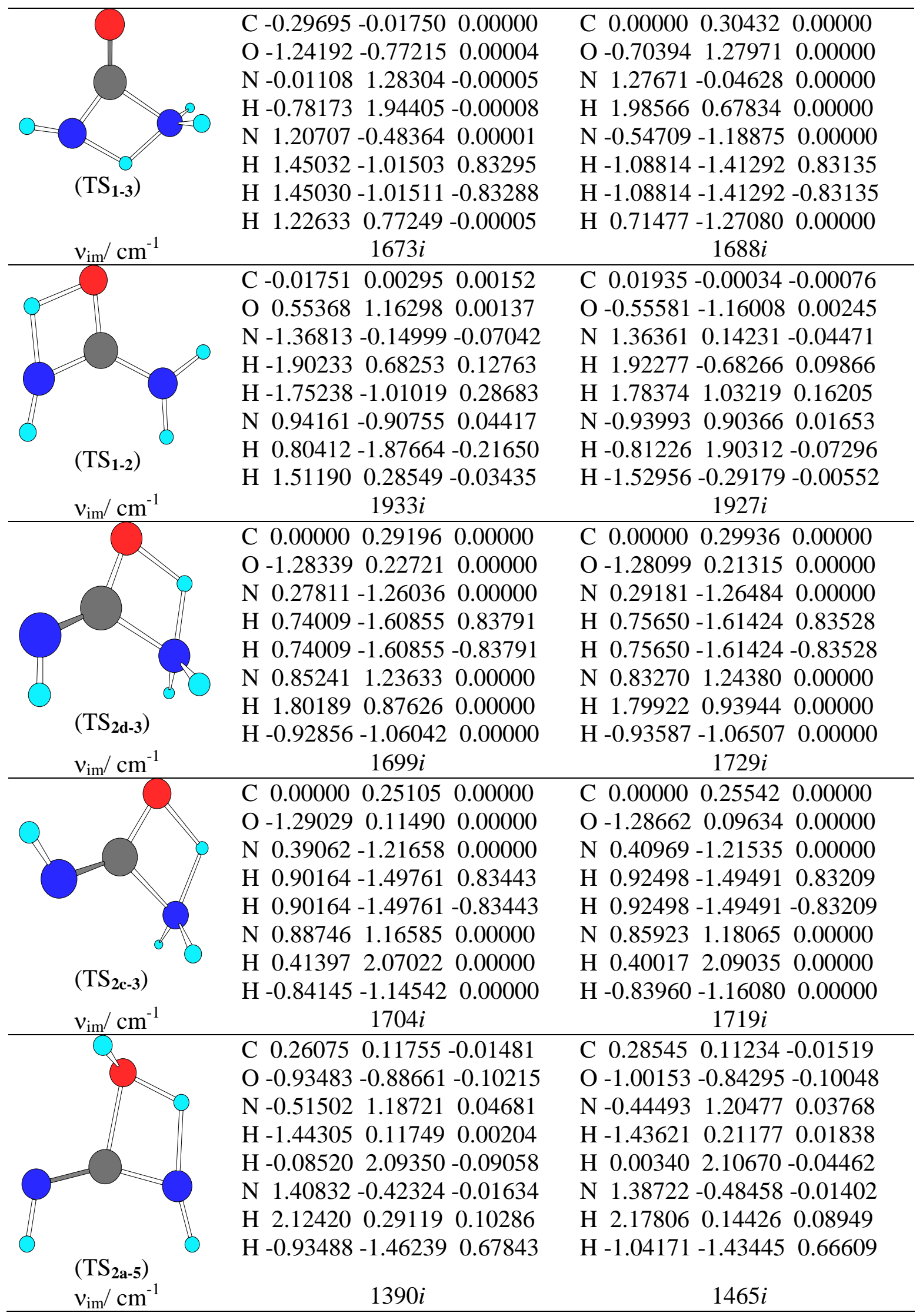




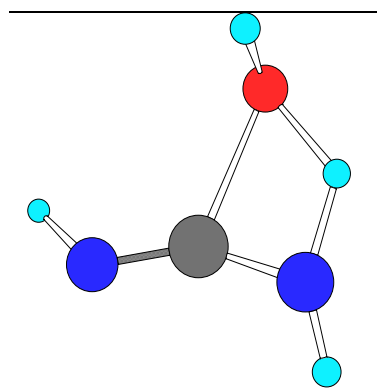

$\left(\mathrm{TS}_{2 \mathrm{~b}-5}\right)$

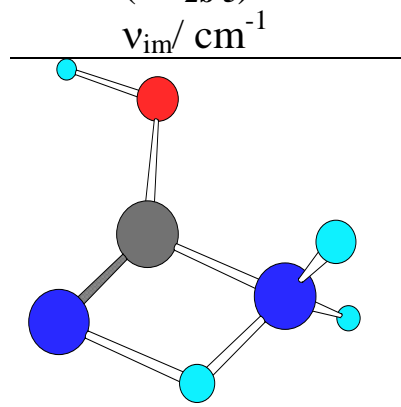

$\left(\mathrm{TS}_{2 \mathrm{a}-4}\right)$

$v_{\text {im }} / \mathrm{cm}^{-1}$

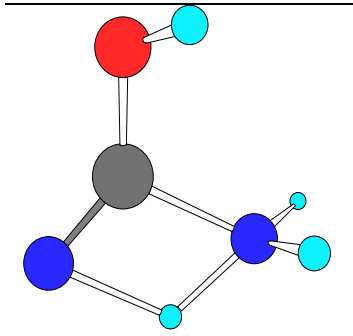

$\left(\mathrm{TS}_{2 \mathrm{~d}-4}\right)$

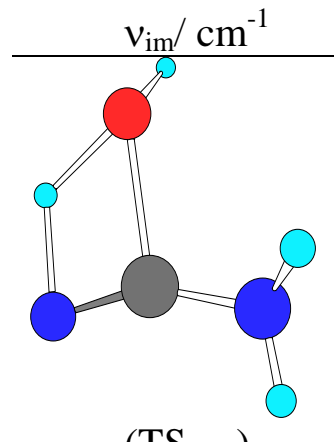

$\left(\mathrm{TS}_{2 \mathrm{c}-6}\right)$

$v_{\text {im }} / \mathrm{cm}^{-1}$
C $0.357870 .14616-0.00100$

O -1.10865 -0.84823 -0.10941

$\mathrm{N}-0.40072 \quad 1.210570 .05894$

$\mathrm{H}-1.34740 \quad 0.33841-0.04779$

H $0.002522 .08589-0.25471$

N $1.50098-0.340150 .04936$

H $1.62520-1.28361-0.29422$

H -1.26021 -1.32477 0.71984
C 0.418040 .138860 .00076

O -1.23126 -0.77122 -0.10778

N -0.29438 1.219920 .05364

H -1.30058 $0.49690-0.00990$

H $0.113282 .09682-0.24703$

N $1.49057-0.447940 .05440$

H $1.60265-1.37679-0.32368$

H -1.44681 -1.28420 0.68203

\section{$1702 i$}

C -0.15558 0.196960 .00000

O -1.23999 -0.63449 0.00000

N $1.15711-0.602340 .00000$

H $1.33578-1.15728-0.83829$

H $1.33578-1.157280 .83829$

N $0.09800 \quad 1.412020 .00000$

H $1.397570 .58085-0.00000$

H -2.00149 -0.03990 0.00000

$1739 i$

C 0.196960 .251550 .02018

O $1.36603-0.43576-0.06555$

$\mathrm{N}-1.14334-0.67963-0.01486$

H -1.36762 -1.22262 0.82033

H -1.29477 -1.23828 -0.85662

$\mathrm{N}-0.193551 .418740 .01813$

H -1.38413 $0.51317-0.02666$

H 1.29475 -1.24923 0.44333

$1836 i$

C $0.215920 .30923-0.00506$

O -1.14091 -0.82524 -0.09533

N $1.36800-0.414820 .08629$

H $1.36976-1.24608-0.48926$

H $2.189740 .16425-0.04278$

$\mathrm{N}-0.384621 .36953-0.00044$

$\mathrm{H}-1.329780 .44157-0.01679$

H -1.28160 -1.29619 0.74093
$1701 i$

C 0.153100 .193160 .00000

O $1.24413-0.630370 .00000$

$\mathrm{N}-1.16358-0.594790 .00000$

H -1.34740 -1.15359 0.83508

H -1.34740 -1.15359 -0.83508

$\mathrm{N}-0.09233 \quad 1.399820 .00000$

$\mathrm{H}-1.402670 .606320 .00000$

H $2.01719-0.05031-0.00000$

C 0.190290 .243830 .02102

O $1.36835-0.42655-0.07279$

$\mathrm{N}-1.14608-0.67600-0.01448$

H -1.36852 -1.22611 0.81637

H -1.29809 -1.23666 -0.85461

N-0.19506 1.404620 .01973

$\mathrm{H}-1.400430 .52668-0.02387$

Н $1.36646-1.214820 .48158$ $1829 i$

C $0.272820 .31549-0.00495$

O -1.22823 -0.76335 -0.08957

N $1.36324-0.474380 .07497$

H $1.32636-1.31777-0.47964$

H 2.240330 .029780 .02450

$\mathrm{N}-0.309761 .36643-0.00350$

$\mathrm{H}-1.304260 .549210 .00958$

H -1.44782 -1.29171 0.69154 
TABLE S2. Optimized Geometries (Cartesian Coordinates/ $\AA$ ) of the Urea Nitrate Conformations and Conformational Transition States.

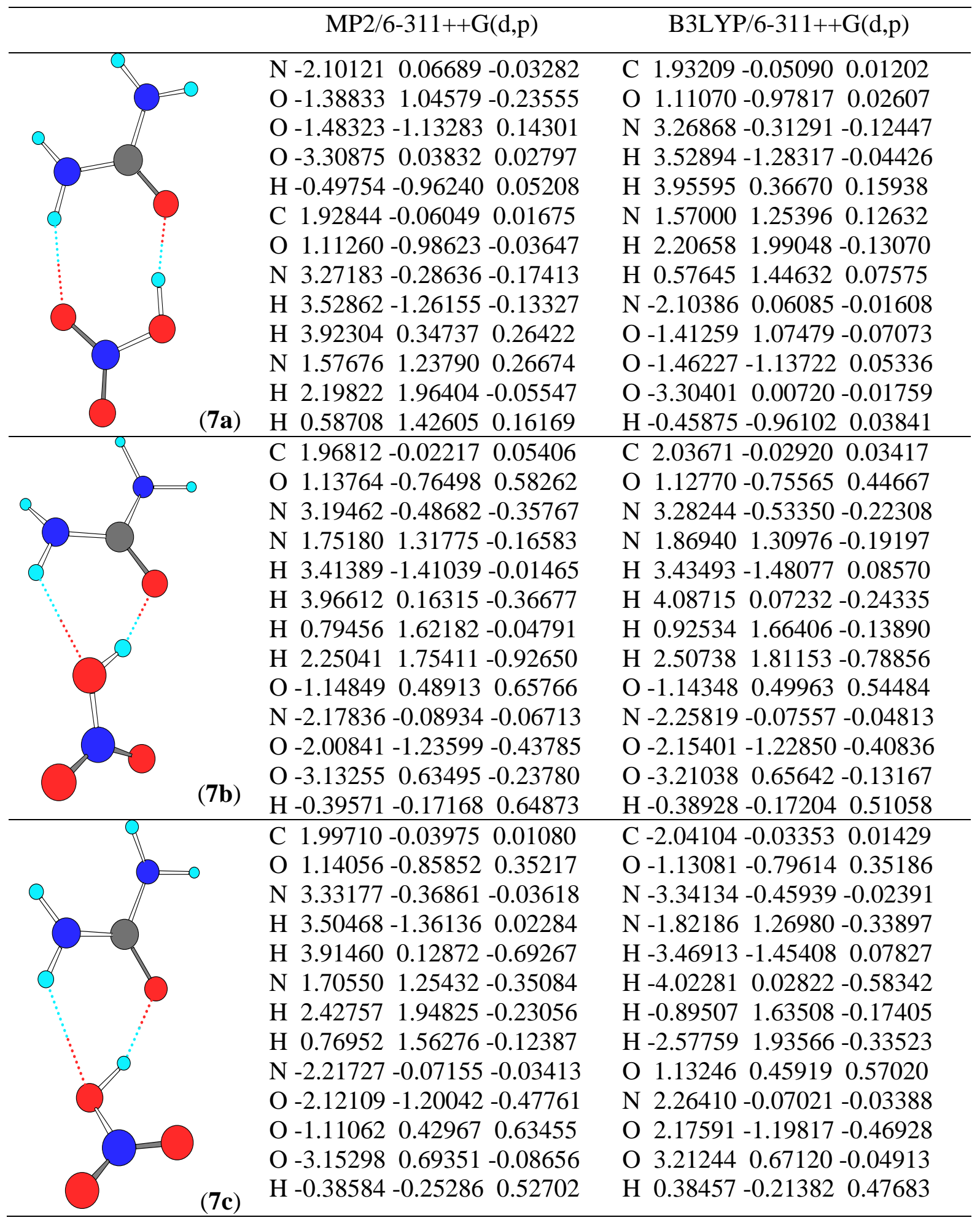




\begin{tabular}{|c|c|c|}
\hline$(8 b)$ & $\begin{array}{lrrr}\mathrm{C} & -2.01179 & 0.01646 & -0.04484 \\
\mathrm{O}-2.92623 & -0.47605 & -0.67295 \\
\mathrm{~N} & -1.19609 & -0.75562 & 0.83731 \\
\mathrm{H}-1.67116 & -1.62973 & 1.04129 \\
\mathrm{H}-0.98005 & -0.25978 & 1.69801 \\
\mathrm{~N}-1.63600 & 1.33556 & -0.08657 \\
\mathrm{H}-0.67257 & 1.55597 & 0.12911 \\
\mathrm{H}-2.05673 & 1.86225 & -0.83789 \\
\mathrm{~N} & 1.98359 & 0.03862 & -0.04321 \\
\mathrm{O} & 1.37212 & 0.93237 & 0.53285 \\
\mathrm{O} & 1.30292 & -1.12906 & -0.27985 \\
\mathrm{O} & 3.12530 & 0.05260 & -0.43563 \\
\mathrm{H} & 0.39788 & -0.99623 & 0.13049 \\
\mathrm{C} & -2.17065 & -0.17450 & -0.07198 \\
\mathrm{O} & -2.42467 & -0.98598 & -0.93702 \\
\mathrm{~N} & -1.07868 & -0.33064 & 0.82115 \\
\mathrm{H} & -0.76107 & -1.29575 & 0.80894 \\
\mathrm{H} & -1.29033 & -0.03556 & 1.76930 \\
\mathrm{~N} & -2.88620 & 0.98274 & 0.15382 \\
\mathrm{H} & -2.40014 & 1.76755 & 0.56227 \\
\mathrm{H} & -3.52510 & 1.21867 & -0.59167 \\
\mathrm{~N} & 2.24746 & 0.01187 & -0.04992 \\
\mathrm{O} & 1.95627 & -1.08047 & 0.40971 \\
\mathrm{O} & 1.22925 & 0.94873 & -0.11368 \\
\mathrm{O} & 3.31322 & 0.40209 & -0.46223 \\
\mathrm{H} & 0.42986 & 0.46926 & 0.23355\end{array}$ & 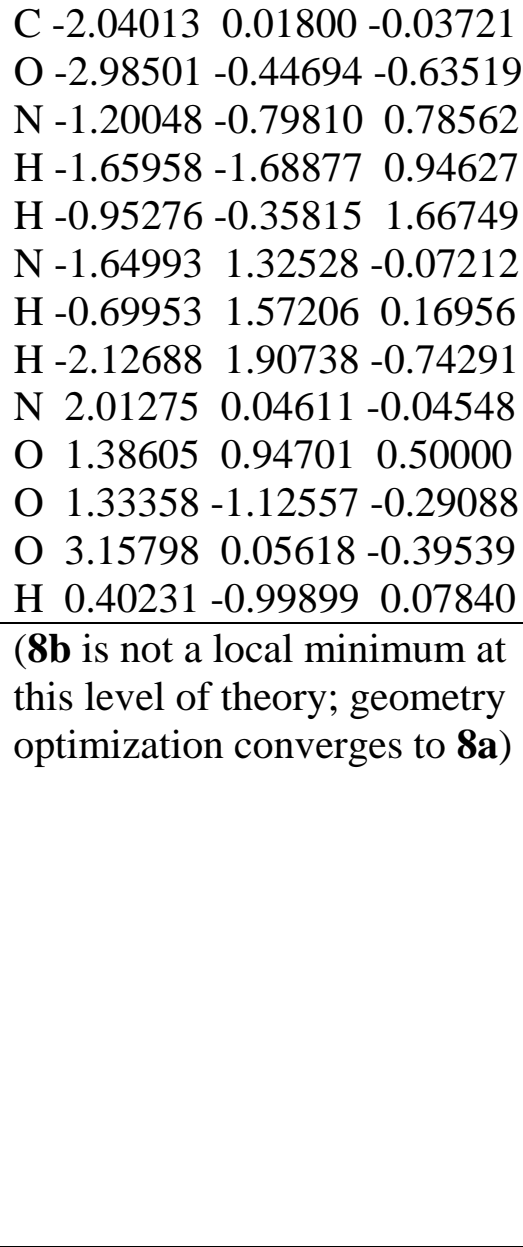 \\
\hline$\left.\underset{-1}{\left(T_{8} \mathbf{a}-\mathbf{b}\right.}\right)$ & $\begin{array}{lrrr}\mathrm{C} & 1.93842 & 0.08131 & -0.07430 \\
\mathrm{O} & 2.36783 & 1.14812 & -0.45773 \\
\mathrm{~N} & 1.32556 & -0.09110 & 1.19989 \\
\mathrm{H} & 1.47687 & 0.74386 & 1.75719 \\
\mathrm{H} & 1.67510 & -0.91157 & 1.68698 \\
\mathrm{~N} & 1.98034 & -1.09358 & -0.79533 \\
\mathrm{H} & 2.22894 & -0.96427 & -1.76576 \\
\mathrm{H} & 1.24495 & -1.76710 & -0.63249 \\
\mathrm{O} & -1.26002 & -0.88179 & 0.56620 \\
\mathrm{~N} & -1.97837 & 0.13179 & -0.06163 \\
\mathrm{O} & -1.48419 & 1.24406 & -0.02867 \\
\mathrm{O} & -3.01422 & -0.23263 & -0.56458 \\
\mathrm{H} & -0.42428 & -0.44059 & 0.87751 \\
\end{array}$ & $\begin{array}{lrrr}\mathrm{C} & -2.08703 & 0.07315 & -0.02770 \\
\mathrm{O} & -2.55996 & 1.14451 & -0.32826 \\
\mathrm{~N} & -1.29724 & -0.68764 & -0.93728 \\
\mathrm{H} & -1.39718 & -0.30036 & -1.86985 \\
\mathrm{H} & -1.51859 & -1.67908 & -0.93744 \\
\mathrm{~N} & -2.24481 & -0.53085 & 1.19390 \\
\mathrm{H} & -2.66062 & 0.04614 & 1.90902 \\
\mathrm{H} & -1.58298 & -1.22425 & 1.50726 \\
\mathrm{O} & 1.42447 & -0.99155 & -0.28397 \\
\mathrm{~N} & 2.12464 & 0.16843 & 0.05065 \\
\mathrm{O} & 1.50433 & 1.20930 & -0.00719 \\
\mathrm{O} & 3.26893 & -0.01622 & 0.36106 \\
\mathrm{H} & 0.50119 & -0.69918 & -0.52679\end{array}$ \\
\hline & $26 i$ & $28 i$ \\
\hline
\end{tabular}




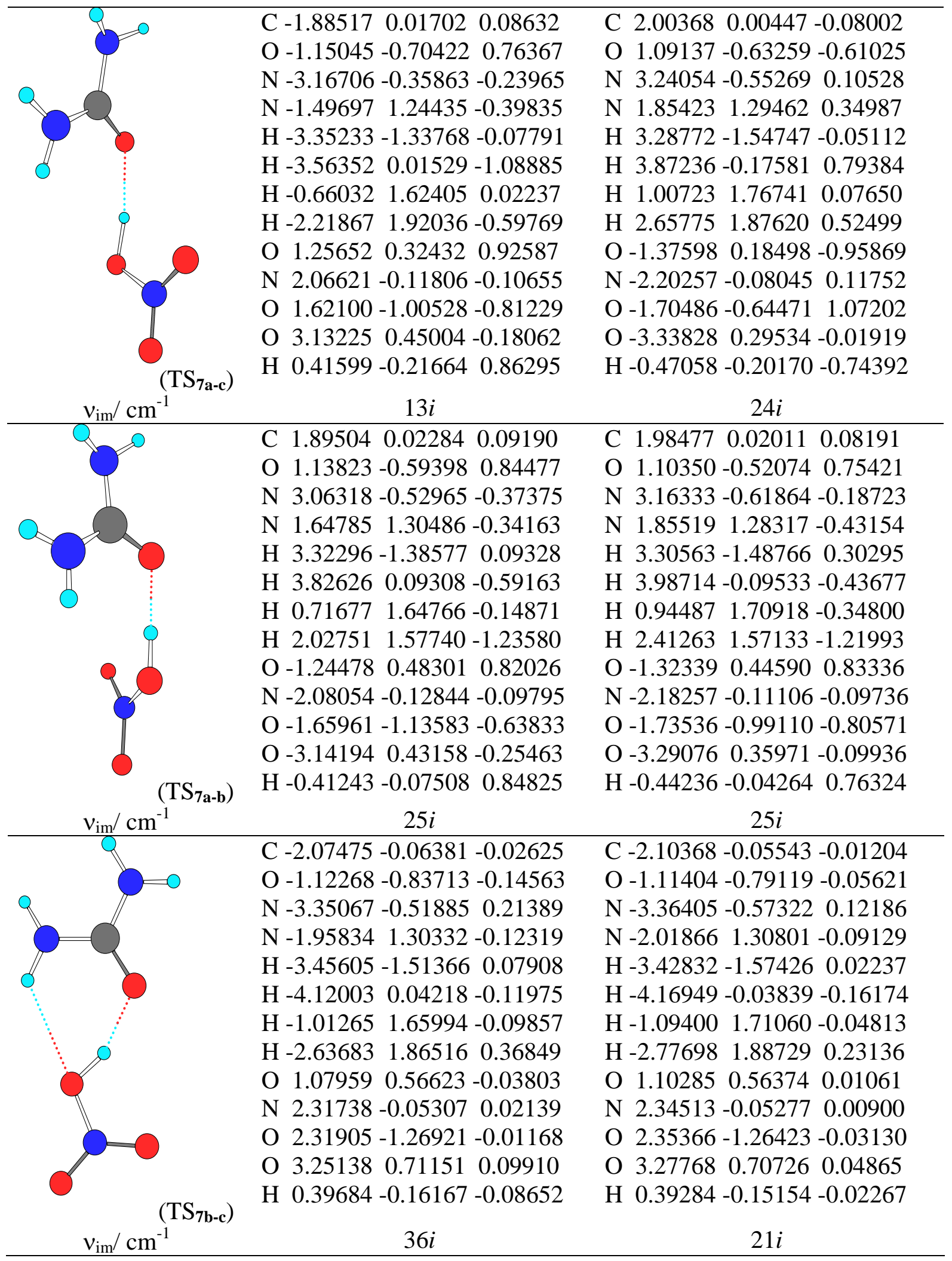


TABLE S3. Optimized Geometries (Cartesian Coordinates/Å) of the Species and Transition States Involved in the UN(g) Transformations.

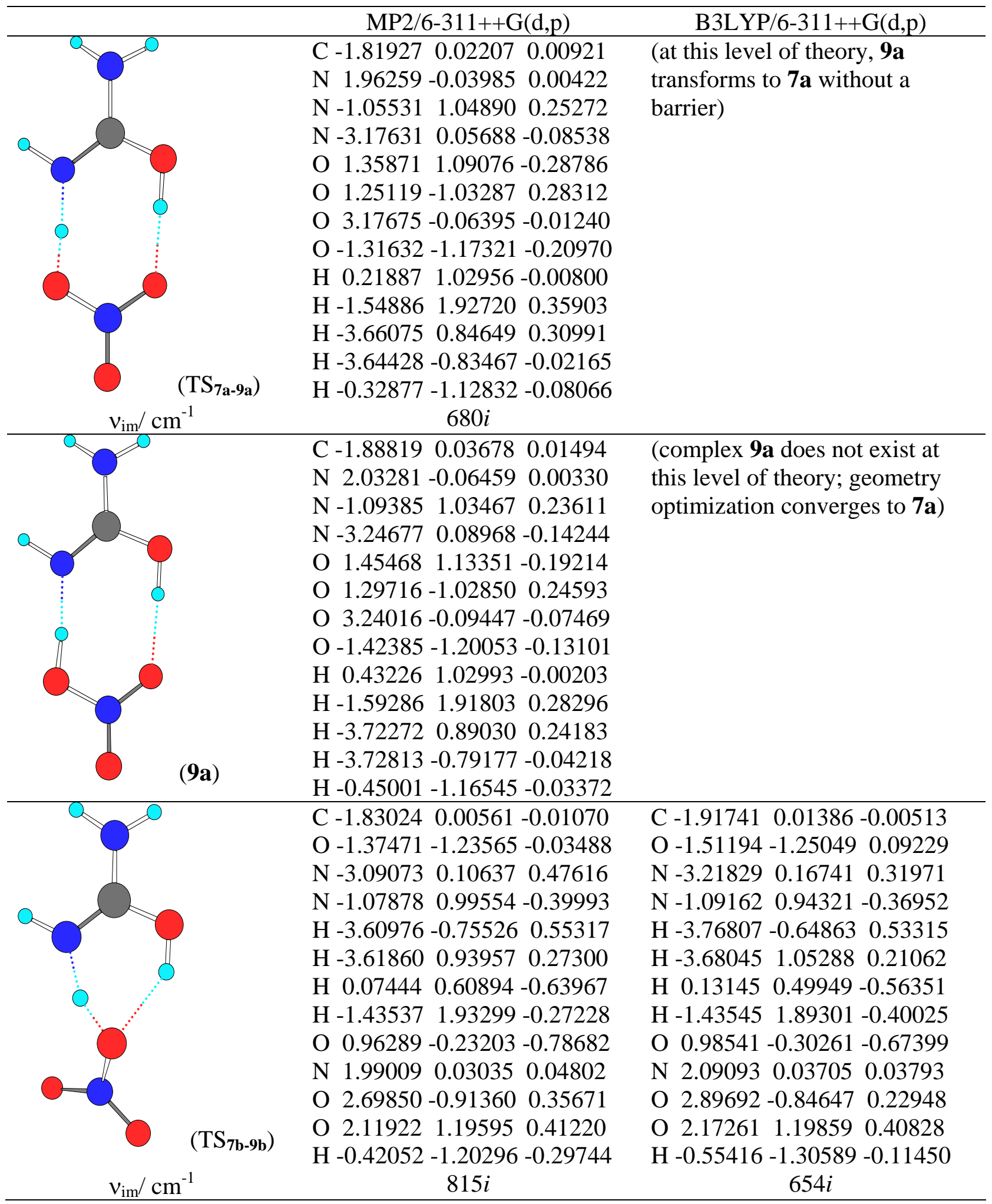




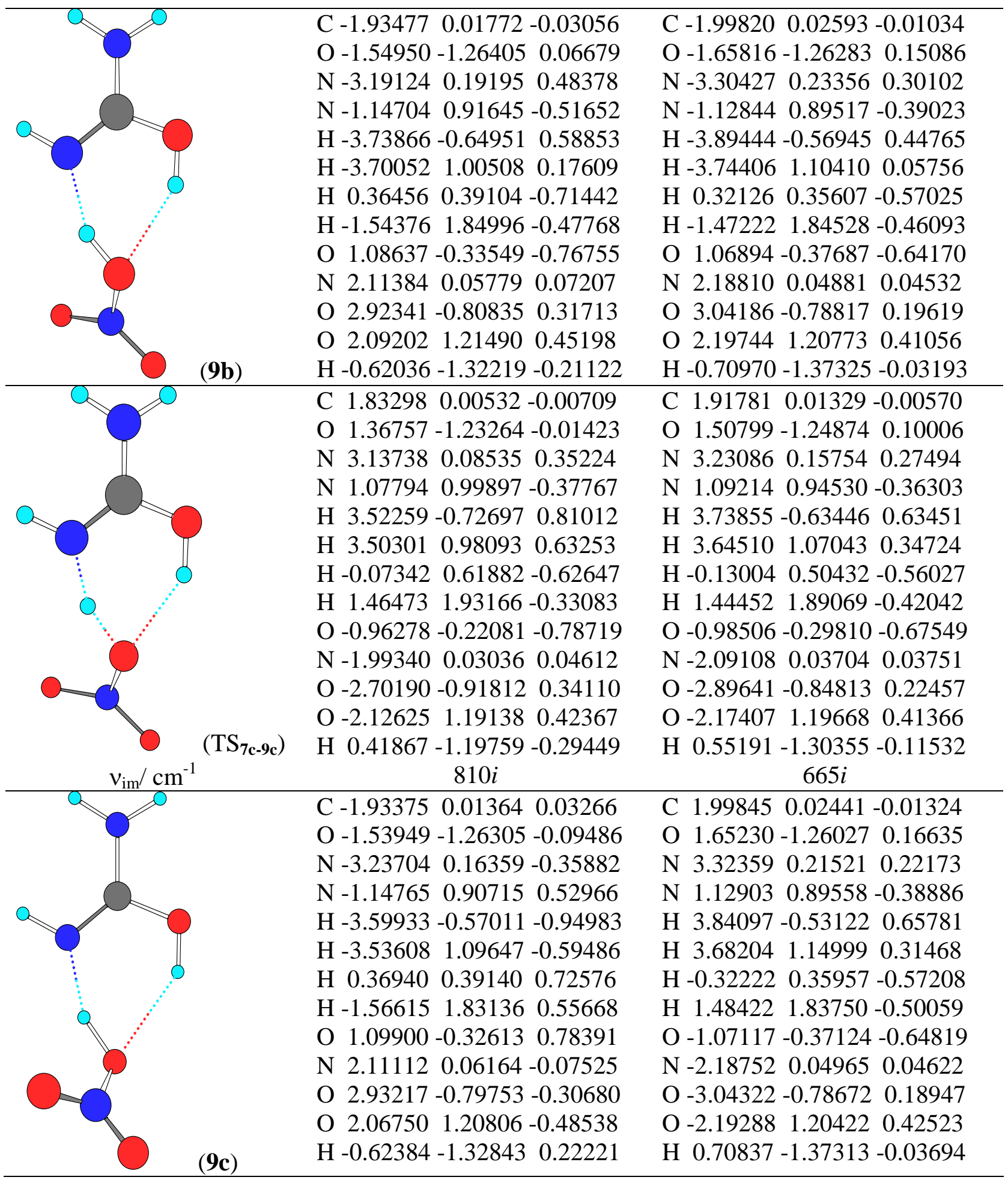




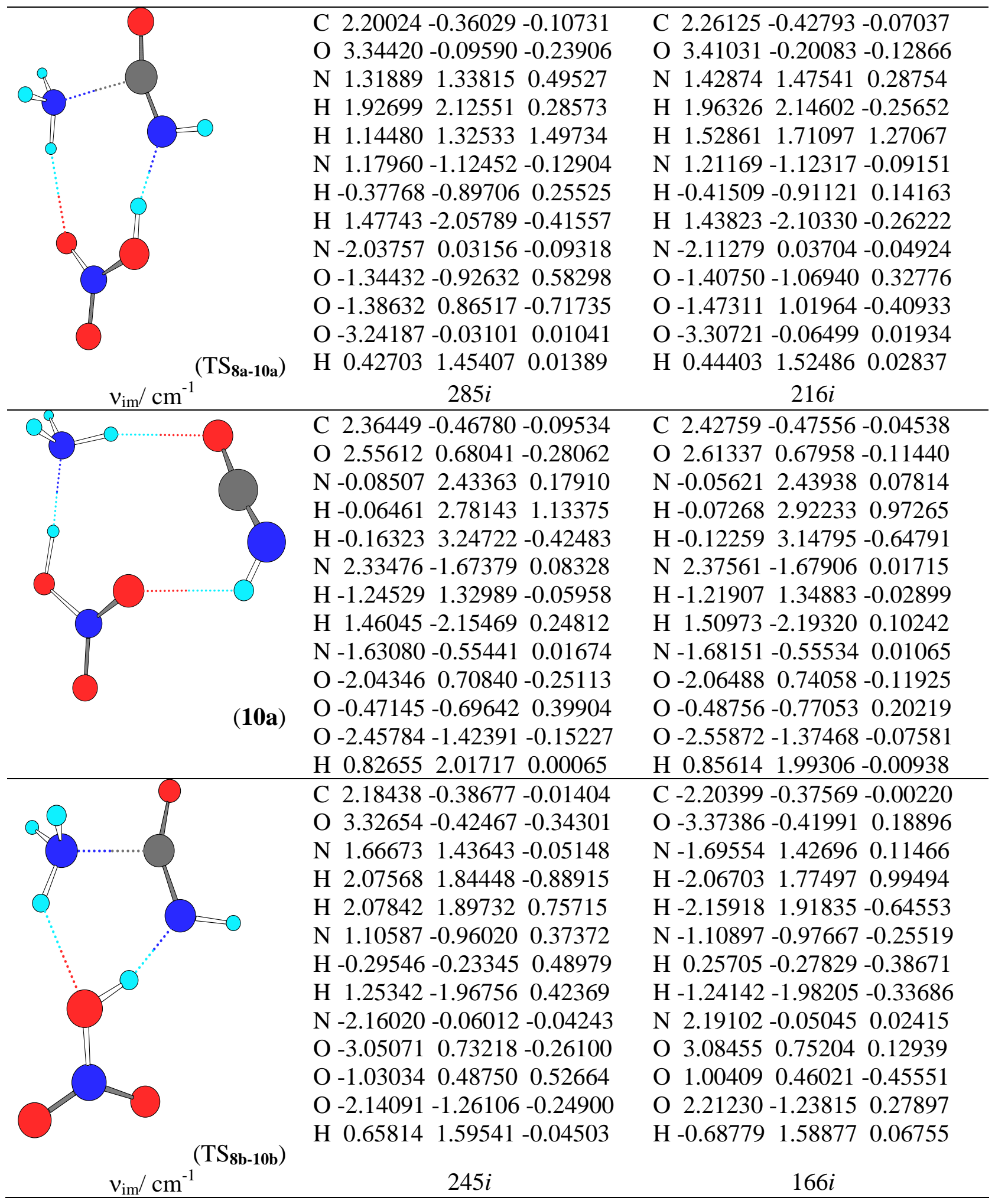




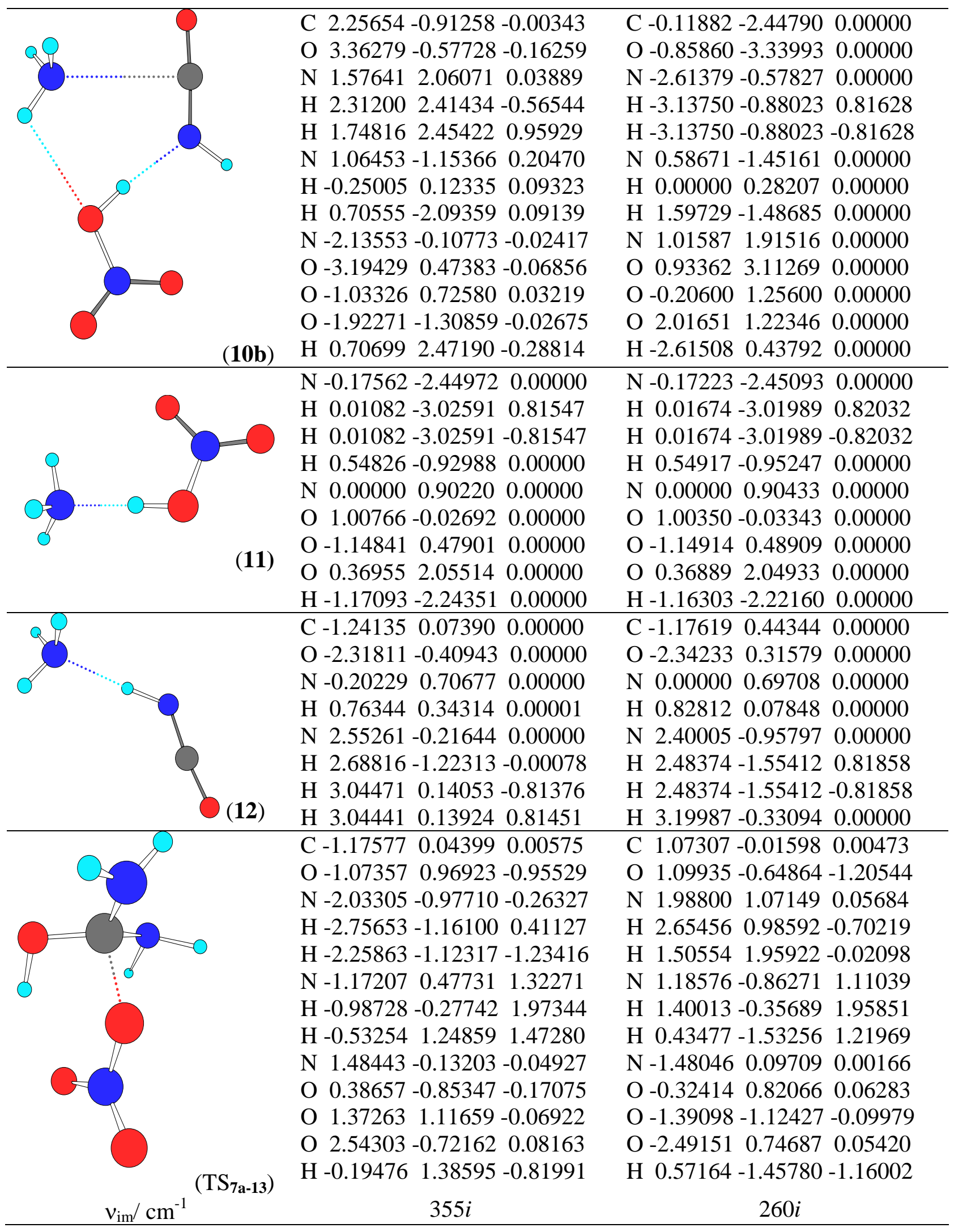




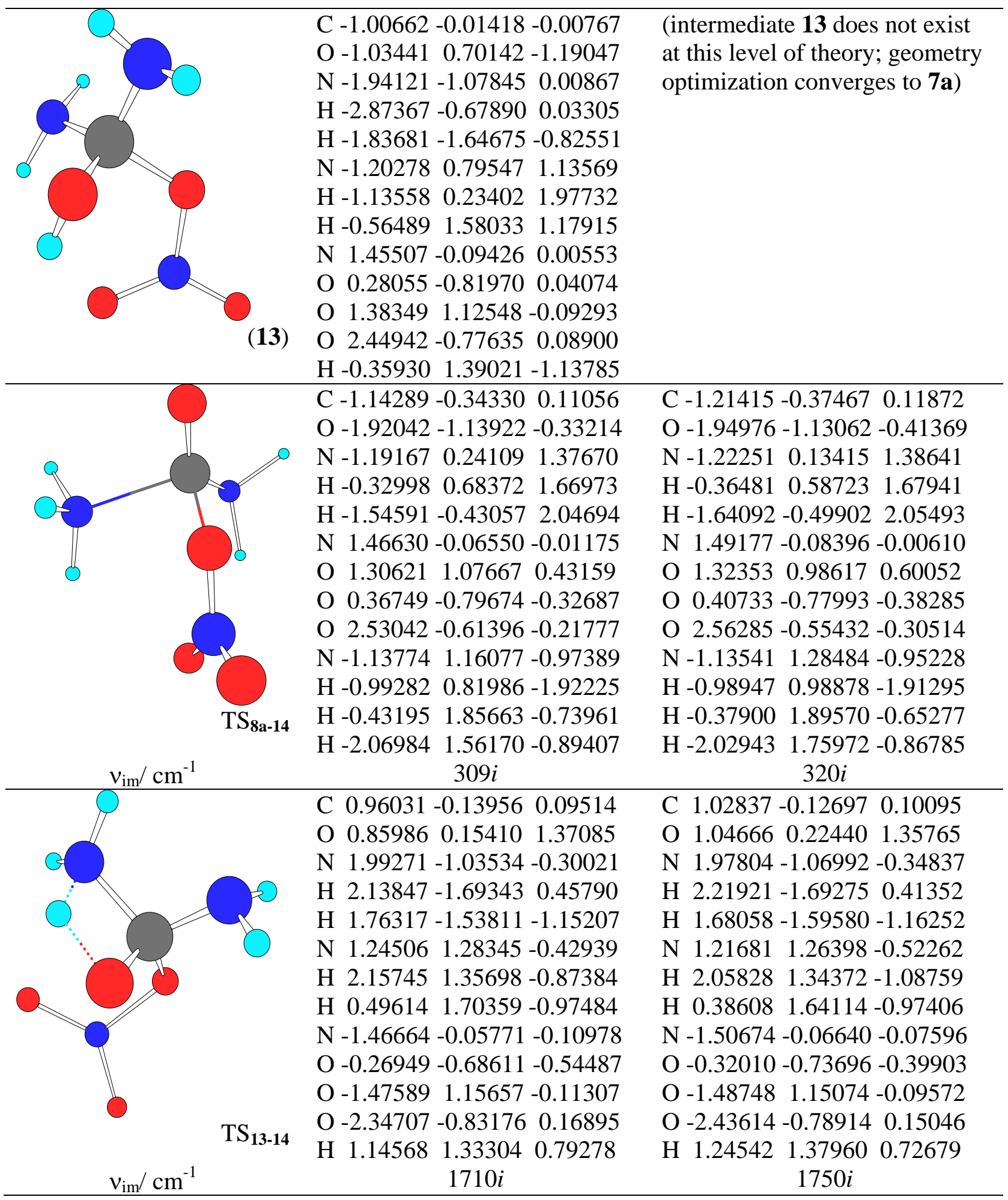




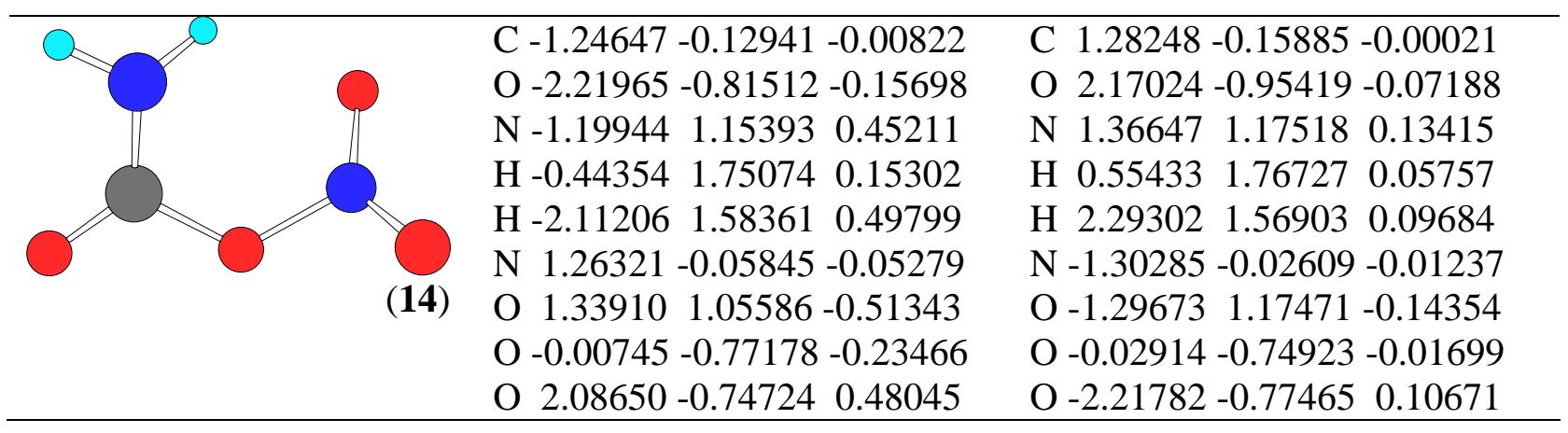


Table S4. Total Energies (ZPE corrected, in Hartree) of Urea and Relative Energies (in kcal/mol, $T=0 \mathrm{~K}$ ) of Urea Isomers and Decomposition Products.

\begin{tabular}{lccccccccc}
\hline $\begin{array}{l}\text { computational } \\
\text { method/basis set }\end{array}$ & Urea (1) & 2a & 2b & 2c & 2d & $\begin{array}{c}\mathrm{NH}_{3}+ \\
\text { HNCO(3) }\end{array}$ & $\begin{array}{c}\mathrm{NH}_{3}+ \\
\mathrm{HOCN}(4)\end{array}$ & $\begin{array}{c}\mathrm{NH}_{3}+ \\
\mathrm{HNCNH}(5)\end{array} \mathrm{H}_{2} \mathrm{NCN}(6)$ \\
\hline B3LYP/6-311++G(d,p) & -225.28638 & 16.17 & 18.21 & 19.96 & 24.35 & 12.81 & 41.34 & 29.85 \\
MP2/6-311++G(d,p) & & & & & & & & 30.21 \\
MP2/6-311G(d,p) & -224.69184 & 15.57 & 17.61 & 19.02 & 23.36 & 12.86 & 36.94 & 30.68 & 25.08 \\
MP3/6-311G(d,p) & -224.67744 & 15.98 & 17.97 & 19.12 & 23.70 & 12.83 & 37.52 & 33.64 & 27.89 \\
MP4(SDTQ)/6-311G(d,p) & -224.72551 & 16.16 & 18.10 & 18.97 & 23.42 & 11.52 & 37.12 & 33.05 & 28.40 \\
CCSD(T)/6-311G(d,p)) & -224.72133 & 15.30 & 17.28 & 17.98 & 22.48 & 13.86 & 37.34 & 33.71 & 28.33 \\
MP2/6-311+G(3df,2p) & -224.82589 & 15.62 & 17.50 & 19.32 & 23.08 & 13.65 & 39.90 & 31.99 & 28.95 \\
G2M(RCC,MP2) & -224.86979 & 14.95 & 16.81 & 18.18 & 21.86 & 14.68 & 39.72 & 32.06 & 29.39 \\
\hline
\end{tabular}

a Calculated at the MP2/6-311++G(d,p) optimized geometries with ZPE corrections obtained at the same level. All other methods use the B3LYP/6-311++G(d,p) optimized geometries and ZPE corrections. 
Table S5. Relative Energies (ZPE-corrected, in kcal/mol, $T=0 \mathrm{~K}$ ) of the Transition States Involved in Urea Isomerization/Decomposition.

\begin{tabular}{|c|c|c|c|c|c|c|c|c|c|c|c|c|c|}
\hline $\begin{array}{l}\text { computational } \\
\text { method/basis set }\end{array}$ & $\mathrm{TS}_{1-2}$ & $\mathrm{TS}_{1-3}$ & $\mathrm{TS}_{2 \mathrm{a}-\mathrm{b}}$ & $\mathrm{TS}_{2 \mathrm{~b}-\mathrm{c}}$ & $\mathrm{TS}_{2 \mathrm{c}-\mathrm{d}}$ & $\mathrm{TS}_{2 \mathrm{a}-\mathrm{d}}$ & $\mathrm{TS}_{2 \mathrm{a}-4}$ & $\mathrm{TS}_{2 \mathrm{~d}-4}$ & $\mathrm{TS}_{2 \mathrm{c}-3}$ & $\mathrm{TS}_{2 \mathrm{~d}-3}$ & $\mathrm{TS}_{2 \mathrm{a}-5}$ & $\mathrm{TS}_{2 \mathrm{~b}-5}$ & $\mathrm{TS}_{2 \mathrm{c}-6}$ \\
\hline /6-311 & 9 & 1 & 36 & 4 & 7 & 5 & 3 & 5 & 6 & 3 & 1 & 7 & 9.43 \\
\hline MP2/6-311++G(d,p) ${ }^{\mathbf{a}}$ & 46.42 & 44.80 & 35.85 & 21.68 & 43.09 & 24.67 & 80.45 & 88.13 & 52.84 & 58.13 & 73.55 & 1.58 & 8.05 \\
\hline AP)/6-311 & 1565 & 44.74 & 37.23 & 2211 & 44.66 & 2515 & 80.79 & 8809 & 52.74 & 58.18 & 73.98 & 73.10 & 79.73 \\
\hline 4P3/ & 5100 & 48.28 & 276 & 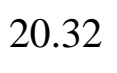 & 44.50 & 2૫ २1 & 83.86 & 91.04 & 56.65 & 62.45 & 78.50 & 80.51 & 36.87 \\
\hline MP4(SDTQ)/6-3 & 45.69 & 40.45 & 37.77 & 22.03 & 45.03 & 24.91 & 80.70 & 87.49 & 52.53 & 57.68 & 74.63 & 72.82 & 79.47 \\
\hline $\operatorname{CCSD}(\mathrm{T}) / 6-311 \mathrm{G}(\mathrm{d}, \mathrm{p}))$ & 47.01 & 45.80 & 37.37 & 20.95 & 44.48 & 23.85 & 80.68 & 87.47 & 53.17 & 58.57 & 75.22 & 74.74 & 81.10 \\
\hline MP2/6-311+G(3df,2p) & 47.25 & 43.16 & 34.63 & 22.00 & 41.65 & 24.77 & 81.95 & 89.25 & 53.32 & 57.61 & 73.67 & 72.27 & 79.62 \\
\hline G2M(RCC,MP2) & 48.60 & 4.22 & 34.77 & 0.85 & 41.47 & 3.47 & 81.84 & 88.62 & 53.75 & 58.00 & 74.91 & 73.91 & 80.99 \\
\hline
\end{tabular}

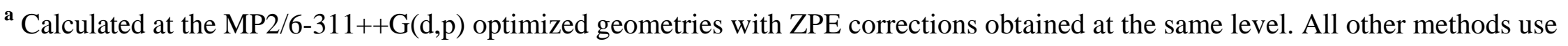
the B3LYP/6-311++G(d,p) optimized geometries and ZPE corrections. 
Table S6. Total Energies (ZPE corrected, in Hartree) of Urea $+\mathrm{HNO}_{3}$ and Relative Energies (in kcal/mol, $T=0 \mathrm{~K}$ ) of the Intermediates Involved in the UN(g) Transformations.

\begin{tabular}{|c|c|c|c|c|c|c|c|c|c|c|c|c|}
\hline $\begin{array}{l}\text { computational } \\
\text { method/basis set }{ }^{\text {a }}\end{array}$ & $\begin{array}{c}\mathrm{NH}_{2} \mathrm{C}(\mathrm{O}) \mathrm{NH}_{2} \\
+\mathrm{HNO}_{3}\end{array}$ & $7 a$ & $7 \mathbf{b}$ & $7 c$ & $8 a$ & $8 \mathbf{b}$ & 9a & 9b & 9c & 10a & 10b & 13 \\
\hline B3LYP/6-311++G(d,p) & -506.23874 & -14.95 & -11.32 & -11.31 & -8.46 & $(-)$ & $(-)$ & 2.15 & 2.17 & -4.14 & 4.51 & $(-)$ \\
\hline BB1K/6-311++G(d,p) & -505.99555 & -15.39 & -11.39 & -11.38 & -8.81 & $(-)$ & $(-)$ & 1.89 & 1.91 & 0.78 & 8.91 & 13.08 \\
\hline MP2/6-311++G(d,p) & -504.99357 & -13.81 & -11.07 & -11.10 & -10.16 & -7.78 & -2.33 & 2.01 & 1.98 & -5.34 & 1.74 & 11.43 \\
\hline MP2/6-311G(d,p) & -504.96377 & -14.88 & -12.02 & -12.07 & -12.14 & $-9.53^{*}$ & -4.04 & $1.05^{*}$ & $(-)$ & -7.65 & 0.58 & $10.34^{*}$ \\
\hline MP3/6-311G(d,p) & -504.94621 & -15.79 & -12.14 & -12.18 & -11.70 & $-8.74^{*}$ & -5.15 & $0.39^{*}$ & $(-)$ & -3.08 & 4.92 & $9.23^{*}$ \\
\hline MP4(SDTQ)/6-311G(d,p) & -505.04963 & -14.25 & -11.45 & -11.50 & -11.96 & $-9.57^{*}$ & -3.26 & $2.10^{*}$ & $(-)$ & -8.20 & -0.44 & $9.62^{*}$ \\
\hline $\operatorname{CCSD}(\mathrm{T}) / 6-311 \mathrm{G}(\mathrm{d}, \mathrm{p}))$ & -505.03208 & -15.07 & -11.85 & -11.91 & -12.10 & $-9.42^{*}$ & -4.50 & $1.03^{*}$ & $(-)$ & -6.35 & 1.62 & $9.12^{*}$ \\
\hline MP2/6-311+G(3df,2p) & -505.29079 & -15.21 & -11.86 & -11.87 & -9.90 & $-7.27^{*}$ & -3.25 & $1.40^{*}$ & $(-)$ & -3.65 & 3.46 & $10.55^{*}$ \\
\hline G2M(RCC,MP2) & -505.35910 & -15.41 & -11.69 & -11.71 & -9.86 & $-7.17^{*}$ & -3.71 & $1.38^{*}$ & $(-)$ & -2.36 & 4.50 & $9.34^{*}$ \\
\hline
\end{tabular}

${ }^{\text {a }}$ Geometry optimization was performed at the B3LYP, BB1K and MP2 levels with the 6-311++G $(d, p)$ basis set. All other calculations were done using the B3LYP/6-311++G(d,p) geometries and ZPE corrections. Values with an asterisk are calculated using the MP2/6-311++G(d,p) optimized geometries and ZPE corrections for those structures, which could not be optimized at the B3L YP/6-311++G(d,p) level. 
Table S7. Relative Energies (in kcal/mol, $T=0 \mathrm{~K}$ ) of the Transition States and Products Involved in the UN(g) Transformations.

\begin{tabular}{|c|c|c|c|c|c|c|c|c|c|c|c|c|}
\hline $\begin{array}{l}\text { computational } \\
\text { method/basis set }{ }^{\mathbf{a}}\end{array}$ & $\mathrm{TS}_{7 \mathrm{a}-9 \mathrm{a}}$ & $\mathrm{TS}_{7 \mathrm{~b}-9 \mathrm{~b}}$ & $\mathrm{TS}_{8 \mathrm{a}-10 \mathrm{a}}$ & $\mathrm{TS}_{8 \mathrm{~b}-10 \mathrm{~b}}$ & $\mathrm{TS}_{7 \mathrm{a}-13}$ & $\mathrm{TS}_{8 \mathrm{a}-14}$ & $\mathrm{TS}_{13-14}$ & $\begin{array}{l}(2 \mathbf{a})+ \\
\mathrm{HNO}_{3}\end{array}$ & $\begin{array}{c}\mathrm{NH}_{3}+\mathrm{HNCO} \\
+\mathrm{HNO}_{3}\end{array}$ & $\begin{array}{l}(\mathbf{1 1})+ \\
\mathrm{HNCO}\end{array}$ & $\begin{array}{l}(\mathbf{1 2})+ \\
\mathrm{HNO}_{3}\end{array}$ & $\begin{array}{l}(14)+ \\
\mathrm{NH}_{3}\end{array}$ \\
\hline B3LYP/6-311++G(d,p) & $(-)$ & 0.65 & 11.57 & 13.75 & 19.60 & 24.91 & 50.92 & 16.17 & 12.81 & 0.42 & 5.82 & 10.37 \\
\hline BB1K/6-311++G(d,p) & $(-)$ & 0.39 & 16.64 & 18.22 & 14.94 & 26.95 & 48.36 & 16.03 & 17.76 & 5.22 & 11.34 & 13.09 \\
\hline MP2/6-311++G(d,p) & -3.80 & 1.88 & 11.59 & 13.30 & 14.26 & 23.35 & 44.35 & 15.57 & 12.86 & 0.60 & 5.40 & 10.15 \\
\hline MP2/6-311G(d,p) & $-5.10^{*}$ & 1.18 & 9.48 & 11.91 & 13.58 & 23.06 & 44.57 & 15.98 & 12.83 & -1.41 & 3.72 & 10.28 \\
\hline MP3/6-311G(d,p) & $-5.63^{*}$ & 1.05 & 14.21 & 17.34 & 12.62 & 29.54 & 48.40 & 14.60 & 17.20 & 3.14 & 9.11 & 14.06 \\
\hline MP4(SDTQ)/6-311G(d,p) & $-3.87^{*}$ & 2.69 & 8.61 & 11.53 & 12.84 & 21.33 & 44.02 & 16.16 & 11.52 & -2.03 & 2.97 & 8.09 \\
\hline CCSD(T)/6-311G(d,p)) & $-5.13^{*}$ & 1.53 & 10.57 & 13.47 & 12.17 & 24.24 & 44.74 & 15.30 & 13.86 & -0.01 & 5.46 & 10.64 \\
\hline MP2/6-311+G(3df,2p) & $-5.18^{*}$ & 0.69 & 13.25 & 14.91 & 13.56 & 23.24 & 44.47 & 15.62 & 13.65 & 1.91 & 7.01 & 9.19 \\
\hline G2M(RCC,MP2) & $-5.21^{*}$ & 1.05 & 14.34 & 16.47 & 12.15 & 24.43 & 44.64 & 14.95 & 14.68 & 3.32 & 8.74 & 9.54 \\
\hline
\end{tabular}

${ }^{\text {a }}$ Geometry optimization was performed at the B3LYP, BB1K and MP2 levels with the 6-311++G $(d, p)$ basis set. All other calculations were done using the B3LYP/6-311++G $(d, p)$ geometries and ZPE corrections. Values with an asterisk are calculated using the MP2/6-311++G(d,p) optimized geometries and ZPE corrections for those structures, which could not be optimized at the B3L YP/6-311++G(d,p) level. 